\title{
ETNOTURISMO: UNA APROXIMACIÓN A LAS OPORTUNIDADES Y AMENAZAS QUE IMPLICA PARA LAS CULTURAS INDÍGENAS
}

\author{
Doris Isabel Acuña Medina* \\ Universidad de La Guajira. Colombia \\ Piedad Felisinda Gañán Rojo** \\ Universidad Pontificia Bolivariana. Colombia \\ Sandra Bibiana Arango Alzate*** \\ Universidad Pontificia Bolivariana. Colombia
}

\section{RESUMEN}

Este estudio, primera aproximación de su tipo realizada a partir del análisis bibliográfico de información científica especializada en la temática, examina potenciales oportunidades y amenazas que enfrenta el etnoturismo a nivel mundial, que deben ser consideradas cuyo se desee promover actividades de transferencia, apropiación de tecnología o innovación, porque se constituirán bien sea en vectores de cambio o en elementos a superar. Debates del tema serían importantes para que micro y pequeñas organizaciones étnicas sean competitivas logryo cambios del sistema con equidad y justicia social.

Palabras clave: turismo, etnoturismo, innovación, oportunidades, amenazas.

Fecha de recepción: 14 de octubre de 2017

Fecha de aceptación: 16 de febrero de 2018

"Facultad de Ciencias Económicas y Administrativas. Universidad de La Guajira. 440001 Riohacha. LA GUAJIRA (Colombia).E-mail: dorisisabel@ uniguajira.edu.co

**Facultad de Ingeniería Química, Universidad Pontificia Bolivariana. 050004 Medellín, ANTIOQUIA (Colombia).E-mail: piedad.ganan@upb.edu.co

***Facultad de Ingeniería Industrial, Universidad Pontificia Bolivariana. 050004 Medellín, ANTIOQUIA (Colombia).E-mail: bibiana.arangoa@gmail.com 


\title{
Ethnotourism: an approach to the opportunities y threats that it implies for indige- nous cultures
}

\begin{abstract}
This study, the first approach of its kind based on the bibliographic analysis of specialized scientific information on the subject, examines potential opportunities or threats facing ethnotourism worldwide, which should be considered when promoting transfer activities, technology appropriation or innovation, because they will be constituted either in vectors of change or in elements to overcome. Debates on the theme would be important for micro y small ethnic organizations to be competitive, achieving systemic changes with equity $y$ social justice.
\end{abstract}

Keywords: tourism, ethnotourism, innovation, opportunities, threats.

\section{INTRODUCCIÓN}

Con el paso de los años y favorecido por el uso extendido de nuevas tecnologías que han acortado las distancias y hacen posible a diferentes segmentos del mercado satisfacer necesidades de productos y servicios, el turismo se ha convertido en uno de los principales sectores de la economía al diversificar y facilitar a viajeros el acceso de manera masiva a lugares promocionados o reconocidos como destinos turísticos. Específicamente, entornos megadiversos ${ }^{1}$ donde se encuentra pluralidad cultural, forma de vida tradicional y contemporánea de pueblos indígenas, biodiversidad, diversidad de lugares y paisajes naturales, bienes históricos, culturales y ambientales que constituyen el patrimonio de comunidades étnicas, representan la gran atracción para viajes de ocio moderno y pueden considerarse el detonante de lo que se ha denominado "turismo indígena", "turismo aborigen", "turismo comunitario" y "turismo étnico o etnoturismo" términos empleados para referirse esencialmente a los mismos aspectos.

El desarrollo de esta actividad es visto como oportunidad porque la Organización Mundial de Turismo (OMT) una de sus promotoras, le considera uno de los mecanismos que posibilita el más amplio reparto de los beneficios económicos y sociales, por tanto, uno de los instrumentos más potente para erradicar la pobreza en el mundo (Cox, 2009). Al respecto, grupos étnicos ha mostrado motivación a explorar, recuperar e interpretar los aspectos tradicionales de su identidad local, lo que favorece la comprensión y el establecimiento de una relación más equitativa entre población indígena como anfitriones y sus visitantes, posibilita la demostración de responsabilidad con nosotros mismos y con los demás, también, el entendimiento entre culturas, la empatía y la paz. De esta forma, hace

1 El programa de las Naciones Unidas para el Medio Ambiente, (PNUMA, 2015) ha identificado entornos megadiversos en diferentes regiones, entre ellas, en el sureste asiático (China, Filipina, India, Indonesia y Malasia), en el continente africano (Madagascar, República Democrática del Congo y Sudáfrica), en Oceanía (Australia y Nueva Guinea) y en América (Brasil, Colombia, Ecuador, Estados Unidos, México, Perú y Venezuela), principalmente por ser países que albergan el mayor índice de biodiversidad de la tierra, en conjunto más del 70 $\%$, suponiendo sus territorios el $10 \%$ de la superficie del planeta, por lo que en ellos se adelantan gestiones de cooperación que buscan promover intereses y prioridades comunes relacionadas con la conservación y el uso sostenible de esa diversidad. 
posible que surjan modelos de negocios étnicos aliados para atender mercados objetivos en estilos de gestión de gryes filiales extranjeras con énfasis en el servicio y competidores (Imanishi, 2007).

No obstante, constituido el patrimonio tangible e intangible en el principal atractivo de viajeros e insumo de la actividad etnoturística, su práctica representa una amenaza para entornos megadiversos. Estos generalmente son habitados por población indígena y en la interacción con la población no indígena que llega a visitarle, el intercambio de expectativas, estereotipos y expresiones de identidad y cultura genera transacción de dinero y evidencia falta de empatía influenciada por políticas neoliberales, neocolonialismo, fundamentalismo, racismo, militarismo, inclusive bullying o acoso a otras personas, ocasionyo consecuentemente desbalance en la oferta de productos y servicios del turismo étnico, por ello, se ha llegado a describir como destructor de la autenticidad cultural y medioambiental.

Las anteriores consideraciones despiertan el interés de organismos internacionales como las Naciones Unidas en el marco de la Organización para la Educación, la Ciencia y la Cultura (UNESCO) ${ }^{2}$ desde donde adelanta gestiones para la protección y preservación de esos entornos. También académicos consideran relevante manifestar la incidencia de procesos macroambientales y locales en el etnoturismo, teniendo en cuenta que bienes y recursos patrimoniales configurados como productos y servicios son ocasionalmente usados, mercantilizados y comercializados por el gobierno, la industria, la sociedad dominante y hasta por otros grupos étnicos e instan una gestión efectiva que coadyuve a minimizar la latente preocupación por la necesidad de equilibrar el uso de lo exótico de los pueblos indígenas como gran atracción, con la protección de su cultura y orgullo étnico. Probablemente, esta condición es el resultado de fallas sistémicas que no favorecen la competitividad del sector. En turismo predominan Pequeñas y Medianas Empresas (PyMES) que se caracterizan por tener bajos indicadores en innovación (Hjalager, 2010). De ahí la importancia de propender la efectividad del desarrollo de la actividad etnoturística, principalmente, gestionyo el fortalecimiento de competencias y habilidades de la población étnica e implantyo planes de mejoramiento que generen significativos cambios organizacionales y beneficios en el contexto local al integrar a las políticas, planes y estrategias los objetivos económicos, sociales, culturales y medioambientales, con visión prospectiva, sostenible y sistémica que favorezca la puesta en marcha de un proceso innovador.

La innovación se asocia a dinámicas económicas y sociales responsables por la producción y transformación del conocimiento científico y tecnológico en riqueza económica, bienestar social y desarrollo humano (Robledo, 2013). Se produce cuyo las organizaciones desafían supuestos existentes y formas de pensar que dan lugar a gryes avances o pequeñas adaptaciones de procesos, productos y servicios. Su impulso en turismo étnico implica tener en cuenta que en el sector predominan pequeños empresarios que carecen y tienen limitaciones para este tipo de conocimiento, además, en la actividad se establece vínculos

2 La UNESCO Mantiene desde 1970 el programa "Hombre y Biosfera", de importancia decisiva para el estudio de la interrelación de la acción humana y su entorno natural y bajo su impulso tuvo lugar el Convenio de Ramsar sobre zonas húmedas y el Convenio sobre la Protección del Patrimonio mundial, cultural y natural. Dentro de sus acciones se encuentra la declaratoria de Patrimonio de la Humanidad a lugares que se encuentran en peligro y están limitadas en el financiamiento para su mantenimiento, protección y conservación. 
solo con clientes y se han ignorado las relaciones que se pueden establecer con los otros grupos de interés ${ }^{3}$ locales, fundamentales para que fluya el conocimiento. No obstante, desde la perspectiva en la que la experiencia es un atributo importante para obtener valor estratégico en el proceso de innovación, la disposición y capacidad demostrada por la población indígena como nuevos actores del sector turístico puede ser fundamental para la eficiencia del sistema. Para ello, se requieren relaciones coordinadas y de cooperación intra- y entre organizaciones que pueden ser a través de documentos, conversaciones y uso de elementos simbólicos a partir de las cuales, surjan nuevas ideas que, implementadas y transformadas generan valor. Asimismo, las políticas diseñadas deben ser responsables con el contexto local y orientar la búsqueda de la sostenibilidad, de modo que el flujo de conocimiento resultado de la interacción social entre la población indígena y no indígena sea factor determinante para fortalecimiento de las capacidades organizacionales, favorezca la comprensión del proceso de innovación y el mejoramiento de la calidad de vida en entornos megadiversos.

Muchas comunidades indígenas alrededor del mundo carecen de calidad de vida (El Mundo Indígena, 2015). Aprovechan la abundancia de recursos y bienes patrimoniales para ofertar etnoturismo como medio para garantizar la sobrevivencia familiar. Al hacerlo, satisfacen necesidades en el presente, propenden la preservación y conservación de su patrimonio, al tiempo, no comprometen la capacidad para que sus futuras generaciones satisfagan las suyas. Sobre la base de la resource-based view ( $r b v)$ la cual enfatiza que una precondición para lograr retornos superiores de manera sostenible, es poseer un conjunto de recursos no disponibles para todas las organizaciones y lograr combinarlos en términos de capacidades, las mismas con las que se obtienen retornos superiores sostenibles en la medida en que sean específicas, valoradas por los clientes, no sustituibles y difíciles de imitar (Barney, 1991; Peteraf, 1993; Rugman y Verbecke, 2004, citados por Dávila, 2012), en el actual entorno globalizado debería promoverse el desarrollo de diferentes capacidades en organizaciones etnoturísticas, para coadyuvar a incrementar su competitividad realizyo actividades más complejas mediante las cuales puedan gestionar transferencia, apropiación de tecnología e innovación y logren cambios en el sistema con equidad y justicia social.

Literatura de diferentes épocas, fuentes y países, ha permitido inferir que ineficientes procesos macroambientales y del contexto local son potencialmente un riesgo para población nativa que habita en entornos megadiversos y ofertan etnoturismo. Aunque diversas aportaciones conceptuales y teóricas sobre transferencia, apropiación de tecnología e innovación están disponibles, estudios del tema se han orientado prácticamente hacia las gryes empresas de la industria del turismo, es decir, no se ha dada la importancia requerida con el propósito de contribuir a que micro y pequeñas organizaciones étnicas sean competitivas en la actualidad.

Sobre la base de las consideraciones anteriores, este estudio, la primera aproximación realizada de su tipo, es uno de los productos del análisis bibliográfico de información científica especializada en el tema. Plantea implicaciones de la práctica del turismo étnico para las comunidades indígenas. Inicialmente se presentan los conceptos base de trabajo para a partir de allí mostrar los aspectos que representan oportunidad o amenaza para la

3 Grupos que tienen interés en las actividades de la organización porque afectan o son afectados por el logro de los objetivos organizacionales (Wheelen y Hunger, 2012). 
industria turística, en particular lo que constituye para las micro y pequeñas organizaciones el ejercer la actividad etnoturística.

\section{ETNOTURISMO E IMPLICACIÓN DE BIENES PATRIMONIALES E INNOVACIÓN EN EL DESARROLLO DE LA ACTIVIDAD}

El desequilibrio con el que se ha venido desarrollado el etnoturismo como actividad económica en el actual entorno globalizado ha tenido significativas implicaciones para comunidades indígenas. Una serie de investigaciones y debates han logrado establecer que este tipo de turismo no es una modalidad turística más como lo son el turismo de aventura o el turismo de salud, entre otros; sino que se trata de nuevos modelos autogestionados de administración turística que principalmente implican: a) una democratización en el manejo de los emprendimientos y negocios turísticos; b) una distribución más equitativa de los beneficios económicos y sociales del turismo; c) una fuente extraordinaria de empleo e ingresos para las familias y la comunidad; d) una alternativa económica basada en el patrimonio cultural y natural, cuya conservación y puesta en valor permite implementar un modelo de desarrollo sostenible con identidad; e) un instrumento que coadyuve a la consolidación y gestión integral de los territorios indígena-campesino-originarios; y f) una estrategia que evite la migración creyo oportunidades y calidad de vida en las propias comunidades (Cox, 2009).

Teniendo en cuenta que los propios turistas a través de su presencia y expectativas influyen en la actividad etnoturística no sólo estimulyo a anfitriones sino también a través de su deseo de exploración cultural, participación y co-creación de productos y experiencias, se podría impulsar la creatividad comercial con ímpetu en las esferas económicas y culturales redescubriendo, reinventyo, importyo y hasta creyo productos culturales y entretenimiento de consumo. En razón a ello, se reconocen impactos positivos concernientes a beneficios socioeconómicos con lo que disminuye la desigualdad regional y se procura la conservación de los recursos naturales y culturales. No obstante, también se observan consecuencias negativas como la destrucción cultural, medioambiental y explotación económica, llegyo incluso a generar preocupación el hecho que la población étnica en el desarrollo de la actividad estaría vendiéndose a sí misma.

El mejoramiento de este escenario convida a promover en las micro y pequeñas organizaciones predominantes en la oferta de productos y servicios étnicos procesos para el desarrollo de capacidades con las que además de innovar, visionen con enfoque de equidad y justicia social el uso del exotismo del patrimonio cultural, tangible e intangible, que atrae a viajeros y se ha configurado en el principal insumo de la actividad. La definición de los conceptos que fundamentan su práctica y que se comentan a continuación, constituyen una aproximación a los retos que tiene el turismo étnico, enfocado específicamente en la población indígena ahora considerados actores de gran importancia en la industria turística.

\subsection{Etnoturismo}

Es una actividad que surge a partir de la incorporación de la población indígena como nuevos actores en la industria turística. Se impulsó con la apertura de mercados por lo que demya comprensión y conciencia en las decisiones tomadas al interactuar entre cul- 
turas, con la naturaleza, la historia y el patrimonio. En esa interacción la población local es influenciada por los visitantes y del mismo modo visitantes pueden ser influenciados y alterados por sus experiencias de viaje (Richards, 2014).

El primer uso del término 〈>turismo étnico〉 se atribuye a Smith (1977) quien lo define como la comercialización al público de las costumbres de pueblos indígenas a menudo exóticas y populares. Para Ceballos (1998) es

"Aquella forma de turismo especializado y dirigido, que se desarrolla en áreas de influencia
de grupos originarios, que poseen un atractivo natural especial y se enmarca en los paráme-
tros del desarrollo humano sostenible. Busca la recreación, el esparcimiento y la educación
del visitante a través de la observación, el estudio de los valores naturales y de los aspectos
culturales relacionados con ellos. Por lo tanto, el turismo étnico es una actividad controlada
y dirigida que produce un mínimo impacto tanto de los ecosistemas naturales como de las
poblaciones autóctonas que viven en función de estos, respeta el patrimonio cultural, educa
y sensibiliza a los actores involucrados acerca de la importancia de conservar la naturaleza".

Tal como se ha mencionado antes, la Organización Mundial de Turismo (OMT) promueve este turismo como uno de los mecanismos que permita el más amplio reparto de los beneficios económicos y sociales y como uno de los instrumentos más potentes para erradicar la pobreza en el mundo. En el nuevo milenio se ha definido como una actividad motivada por la búsqueda de experiencias culturales exóticas que incluye la visitas a pueblos de minorías étnicas, sus casas y parques temáticos, con posibilidad de participar en eventos como festivales, bailes, ceremonias tradicionales o simplemente para comprar artesanía y recuerdos étnicos. El desarrollo de la actividad algunas veces es controlado por la población étnica y también población no indígena utiliza el patrimonio local para atraer viajeros en beneficio particular, razón por la que se ha llegado a tildar de destructor de la autenticidad cultural y medioambiental.

La revisión literaria admite pensar el etnoturísmo como elemento clave en procesos de investigación y desarrollo experimental (I+D), por ello en la tabla 1, se presentan diferentes enfoques desde los cuales se ha estudiado esta actividad económica.

Tabla 1

ENFOQUES DE LA ACTIVIDAD ETNOTURÍSTICA

\begin{tabular}{|l|l|}
\hline \multicolumn{1}{|c|}{ PERSPECTIVAS } & AUTORES \\
\hline $\begin{array}{l}\text { La actividad etnoturística ha motivado a } \\
\text { comunidades indígenas a explorar, recu- } \\
\text { perar y reinterpretar los aspectos tradicio- }\end{array}$ & Stronza y Gordillo, (2008); Cohen, $(1987,1988)$ \\
$\begin{array}{l}\text { nales de su identidad local, lo cual es una } \\
\text { oportunidad como plataforma para que la } \\
\text { población étnica se presente bajo una luz } \\
\text { más positiva }\end{array}$ & \\
\hline
\end{tabular}




\begin{tabular}{|c|c|}
\hline PERSPECTIVAS & AUTORES \\
\hline $\begin{array}{l}\text { La práctica del turismo étnico es potencial } \\
\text { morada para explorar relaciones y respon- } \\
\text { sabilidades con nosotros mismos y con los } \\
\text { demás, fortalecer el entendimiento entre cul- } \\
\text { turas, con manifestación de empatía y paz. }\end{array}$ & $\begin{array}{l}\text { Tucker, (2016); Caton (2012, 2014); Moufakkir y } \\
\text { Kelly (2010). }\end{array}$ \\
\hline $\begin{array}{l}\text { El etnoturismo posibilita la conservación } \\
\text { y revitalización de recursos naturales y } \\
\text { culturales mediante la puesta en marcha } \\
\text { de procesos constructivos en los que se } \\
\text { reviven tradiciones, lenguas y orgullo con } \\
\text { demostraciones artísticas, espectáculos y } \\
\text { festivales. }\end{array}$ & $\begin{array}{l}\text { Yang y Wong, (2012); Yang y Fik, (2014); McIntosh } \\
\text { y Johnson, (2005); Henderson, (2003); Jim, (2000); } \\
\text { Boissevain, (1996). }\end{array}$ \\
\hline $\begin{array}{l}\text { Modelos de negocios étnicos pueden } \\
\text { ser aliados en la atención de mercados } \\
\text { objetivo de filiales extranjeras. Estrategia } \\
\text { socioeconómica con impacto positivo en } \\
\text { comunidades indígenas. }\end{array}$ & $\begin{array}{l}\text { Imanishi, (2007); McIntosh y Johnson, (2005); } \\
\text { Walsh y Swain, (2004); Henderson (2003). }\end{array}$ \\
\hline $\begin{array}{l}\text { El turismo étnico es consecuencia de una } \\
\text { sociedad acomodada, globalizada, acce- } \\
\text { sible y tecnológicamente desarrollada, } \\
\text { que promueve multiplicidad de destinos, } \\
\text { diversificación de productividad, súper } \\
\text { segmentación de la demya, flexibilidad } \\
\text { en la oferta y distribución de productos } \\
\text { y servicios, búsqueda de rentabilidad y } \\
\text { modificación de espacios territoriales. }\end{array}$ & Ibáñez (2005); Zhang y Xiao (2014) \\
\hline $\begin{array}{l}\text { Practicar el etnoturismo causa impactos } \\
\text { negativos al medioambiente, la cultura, a } \\
\text { la forma de vida y el sentido de identidad } \\
\text { de poblaciones indígenas al promover la } \\
\text { explotación económica al usar, comercia- } \\
\text { lizar, mercantilizar, degradar e intimidar } \\
\text { culturas, suplantar identidad de grupos } \\
\text { étnicos y denigrar de los sitios sagrados. }\end{array}$ & $\begin{array}{l}\text { Yang, (2016); Tucker, (2016); Schwarzin, (2012); } \\
\text { Xie, (2011); Ryan y Aicken (2005); Dyer, et al., } \\
\text { (2003); Cohen, (1987, 1988). }\end{array}$ \\
\hline
\end{tabular}

Fuente: Elaboración propia.

En la tabla 1 se observa que en etnoturismo urge un proceso innovador para superar el desbalance en las perspectivas planteados. En atención a ello, la población local debería ser protagonista de su gestión, la población no indígena ser consciente por el impacto de las decisiones tomadas y actuar con respeto, compromiso, sentido de pertenencia y responsabilidad por los bienes patrimoniales que les atrae. Asimismo, se requiere la participación efectiva de los grupos de interés locales. De no ser así, en la industria turística la oferta de productos y servicios a partir de bienes y recursos étnicos representa una amenaza en 
contra de la forma de vida, tradicional y contemporánea, la biodiversidad y diversidad propia de entornos megadiversos reconocidos por el PNUMA.

En consideración a estos planteamientos la gráfica 1 muestra la recopilación de algunos conceptos plasmados en la literatura, en los cuales se considera que la población indígena debe ser el elemento principal de la gestión del etnoturismo, con el propósito de alcanzar óptimos resultados y responder con criterios de equidad y justicia social las oportunidades que ofrece.

\section{Gráfica 1 \\ POBLACIÓN INDÍGENA COMO ELEMENTO PRINCIPAL EN TURISMO ÉTNICO}



Fuente: Elaboración propia.

\subsection{Bienes patrimoniales en la actividad etnoturística}

Patrimonio es un concepto poliédrico con diferentes manifestaciones: cultural, artística, arqueológica, histórica, militar, natural, escénica. Es definido como el territorio que ocupa 
un país su flora, fauna y todas las creaciones y expresiones de las personas que lo han habitado: instituciones sociales, legales, religiosas; lenguaje y cultura material desde las épocas históricas más antiguas (UNESCO, 2003), por ello, son elementos valorados por, y desde diversas circunstancias y enfoques.

Para las comunidades indígenas el fundamento de su vida lo constituyen sus bienes representados en el patrimonio tangible e intangible. En muchos países la reiteración de la identidad cultural basada en las culturas indígenas y en otros grupos locales tradicionales y populares, han posibilitado la construcción de nuevos estados pluriculturales (Leal, 2008). Se reconoce de ellas el eficiente desempeño en su papel de salvaguardia, mantenimiento, producción y recreación del patrimonio cultural, con lo cual han contribuido a enriquecer la diversidad cultural y la creatividad humana. En ese propósito, desde diferentes perspectivas instituciones internacionales realizan acciones que propenden el respeto de los derechos de los pueblos indígenas, entre ellas, La Conferencia Mundial sobre los Pueblos Indígenas, Foro Permanente de la ONU para las Cuestiones Indígenas, El Convenio 169 de la OIT, Convención sobre el Patrimonio Mundial, Objetivos del Desarrollo Sostenible.

El patrimonio cultural es un atributo importante en turismo. Es el gran atractivo de un segmento significativo de viajeros de ocio moderno, lo cual ha contribuido al crecimiento de esta industria de servicio, puede convertirse en un factor de desarrollo económico y mejorar indicadores socioeconómicos. En atención a ello, la tabla 2 muestra algunas acciones con las que se puede lograr una efectiva relación entre patrimonio cultural y etnoturismo, además, en busca de una planificación eficiente del uso y protección de bienes patrimoniales.

\section{Tabla 2 \\ ACCIONES PARA UNA EFECTIVA RELACIÓN ENTRE PATRIMONIO CULTURAL Y ETNOTURISMO}

Implantar técnicas para conseguir cambios de actitudes y comportamientos positivos de viajeros de ocio moderno en referencia al uso adecuado de los bienes y recursos de interés.

Propender una eficiente comunicación entre visitantes y la población visitada para lograr conexión y favorecer la protección, salvaguardia, mantenimiento, conservación y recreación de bienes históricos y culturales que en el nuevo milenio son el principal insumo de la oferta de productos y servicios en la industria turística. a partir de factores étnicos.

Implementación de procesos de difusión y comunicación de lo que significa la existencia de recursos patrimoniales en espacios turísticos para el desarrollo económico, social, cultural, político y ambiental.

Fuente: Elaboración propia.

La orientación de las acciones planteadas en la tabla 2 es al uso eficiente de los bienes que constituyen el patrimonio, tangible e intangible. Turistas e invitados deben mostrar empatía en las comunidades étnicas y los pueblos indígenas deberían liderar la defensa de estos recursos 
de gran significado para sí mismo y para su localidad, teniendo presente que su salvaguardia, protección y mantenimiento contribuye al enriquecimiento de la diversidad cultural.

Indígenas contemporáneos deben estar facultados para participar en la iniciación de medidas de protección, conservación y difusión de su patrimonio cultural. Estos son elementos y valores con los que se reconocen y son reconocidos los grupos étnicos, son base fundamental de su identidad, por lo que resulta inevitable asociar patrimonio e identidad a su conservación. En efecto, estos son en sí la vida misma de las comunidades y producto de su herencia histórica, factores que les diferencian e identifican como cultura, que se renuevan y recrean permanentemente y requieren procesos de valorización, promoción y conservación.

\subsection{Innovación en etnoturismo}

La innovación es el medio por el cual nuevas ideas se transforman o implementan en productos, procesos y servicios que generan valor para organizaciones en el marco de procedimientos que evidencian externalidades de aprendizaje mediatizado tanto por documentos y protocolos como por estructuras verbales e interacciones simbólicas (Javier Castro Spila et al., 2009). En atención a ello, el proceso de innovación en la industria turística conlleva el análisis de factores que pueden ser distintos o compartidos con la de otras industrias de servicios. Aún el sector turístico tiene la oportunidad de aplicar estrategias distintivas en la emergente economía del conocimiento, en la que hay falta de correspondencia entre la naturaleza tangible de las fuentes convencionales de competitividad, e intangibilidad asociada a la información y el conocimiento. Aunque aportaciones conceptuales y teóricas relativas al tema están disponibles, el turismo evidencia fallas sistémicas en gran parte debido a que investigadores y actores políticos y gubernamentales han mostrado más interés en estudiar el tema en las gryes empresas, las que en algunos casos exhiben su gran experiencia y en otros un desempeño desigual con poca evidencia empírica de su eficacia.

Por su parte, las PyMES de turismo son reconocidas por tener bajos niveles de contribución a la innovación, por presentar dificultad para ser innovadoras y porque sus gestores tienen limitaciones para este tipo de aprendizaje. Con esas poderosas razones, rara vez cuentan con departamentos de I+D u otros medios, como por ejemplo la conformación de redes académicas y empresariales para generarle. En ese sentido, el escenario de las micro organizaciones que predominan en la oferta del etnoturismo es muy complejo, precisa colocar en contexto el argumento científico que la innovación se produce cuyo las organizaciones desafían supuestos existentes y formas de pensar que dan lugar a gryes avances o a pequeñas adaptaciones de los procesos, productos y servicios existentes.

El turismo étnico tiene como principal insumo para la oferta de sus productos y servicios el patrimonio tangible e intangible, factores propios de los entornos megadiversos donde se práctica la actividad, relativos a paisajes exóticos, riquezas y diversidad cultural, los cuales son claves para la producción de valor de una manera dinámica e interactiva entre viajeros o turistas, que validan experiencias posteriormente en su memoria, y la población étnica en su papel de anfitriones. Estas particularidades demya de las micro organizaciones etnoturísticas la puesta en escena de habilidades y destrezas para liderar y motivar a comunidades locales a aprovechar las oportunidades socioeconómicas, culturales, políticas y de conservación medioambiental que tienen con la gestión efectiva de la abundancia de 
recursos y bienes que poseen, asimismo, acceder y emplear fuentes externas para producir cambios organizacionales con lo que impacten positivamente en su desempeño, que en gran medida dependen de las capacidades para reconocer el valor de nuevos conocimientos, asimilarlos, transformarlos y explotarlos en esta industria de servicio. Ahora bien, las posibilidades son mayores si se tiene el respectivo acompañamiento del estado en su papel de legislador, planificador, coordinador y regulador, desde donde se apliquen estrategias integradoras e inclusivas, con patrocinios, estímulos, promoción y protección de bienes culturales. Si bien es cierto del estado no se percibe falta de voluntad en el proceso de la innovación, se cuestiona la eficacia de muchos esfuerzos gubernamentales.

Al respecto, se exalta cada vez más la importancia de las políticas de innovación como parte integral. En su ejecución surgen actividades de innovación y desarrollo experimental (I+D) que conllevan a un conocimiento colectivo de áreas de mercados, ciencia y tecnología. De la efectividad de los instrumentos estatales depende la alineación, autorefuerzo y el logro de sus objetivos, también posibilitan eliminar las barreras, proporcionar recursos e incentivos regulatorios, gestionar colaboración del sector público y privado, cofinanciar y estimular la inversión privada. El diseño y aplicación de políticas de innovación en etnoturismo requiere una perspectiva general que tenga en cuenta los conocimientos científicos y saberes empíricos, liderazgo de representantes políticos, gubernamentales y académicos, igualmente, la participación de actores no estatales, incluidas ONG, asociaciones y comunidades minoritarias. Algunos acaecimientos de innovación en estas organizaciones se plasman en la tabla 3.

Ahora bien, el alcance institucional de la innovación desborda por completo el ámbito organizacional y hace hincapié en interdependencia y no linealidad, lo que implica interacción entre instituciones y organizaciones, a través de relaciones complejas propias de estructuras sociales. En este contexto, se implantan Sistemas Nacional de Innovación (SNI) propuestos inicialmente por Lundvall, (1985) y Freeman (1987). Estos han sido ampliamente promovidos y aplicados en escenarios políticos gubernamentales y organizaciones internacionales, como la OCDE y la Unión Europea, siendo articulados a las regiones y localidades para coadyuvar a la distribución y uso del conocimiento, además, de favorecer la conformación de redes internacionales que fomentan el aprendizaje.

Tabla 3

INNOVACIÓN EN INDUSTRIA TURÍSTICA-ETNOTURISMO

\begin{tabular}{|l|l|}
\hline \multicolumn{1}{|c|}{ RESEÑAS DE LA INNOVACIÓN } & \multicolumn{1}{|c|}{ AUTORES } \\
\hline $\begin{array}{l}\text { El proceso de innovación en turismo evidencia falla sis- } \\
\text { témica que no favorece su competitividad, posiblemente } \\
\text { porque investigadores, actores políticos y gubernamentales } \\
\text { han mostrado más interés en estudiar el tema en las gryes } \\
\text { empresas. }\end{array}$ & $\begin{array}{l}\text { Blichfeldt, (2009); Thomas y Wood, } \\
(2014), \text { Hjalager (2010, 2012). }\end{array}$ \\
\hline $\begin{array}{l}\text { Estudios han llegado a colegir que investigaciones sobre el } \\
\text { tema han sido limitada con desempeño desigual y poca evi- } \\
\text { dencia empírica de su eficacia, quizás porque no se enfatiza } \\
\text { el tema en discursos de sistemas de innovación, además, se } \\
\text { cuestiona eficacia de los esfuerzos gubernamentales. }\end{array}$ & $\begin{array}{l}\text { Hall y Williams, (2008); Hall, } \\
\left(2009^{a}\right) ; \text { Hjalager, (2010, 2012). }\end{array}$ \\
\hline
\end{tabular}




\begin{tabular}{|c|c|}
\hline RESEÑAS DE LA INNOVACIÓN & AUTORES \\
\hline $\begin{array}{l}\text { Mejorar la dinámica de la innovación en la industria turística } \\
\text { requiere adoptar una actitud proactiva sobre todo a un cam- } \\
\text { bio ambicioso de observación de la tecnología simple de la } \\
\text { inteligencia económica. }\end{array}$ & Decelle, (2004). \\
\hline $\begin{array}{l}\text { El impulso de innovación en la actividad etnoturística } \\
\text { implica disposición y capacidades de los indígenas para } \\
\text { aprovechar oportunidades económicas con mentalidad hacia } \\
\text { la sostenibilidad. }\end{array}$ & Sakdiyakorn y Sivarak, (2015) \\
\hline $\begin{array}{l}\text { Riqueza y diversidad cultural pueden ser el elemento cen- } \\
\text { tral para la producción de valor de una manera dinámica e } \\
\text { interactiva. }\end{array}$ & Stamboulisa y Skayannis, (2003). \\
\hline $\begin{array}{l}\text { Las pequeñas y medianas empresas (PyMES) de la industria } \\
\text { turística tienen bajos niveles de contribución a la innovación, } \\
\text { rara vez cuentan con departamentos de I+D, escisiones con } \\
\text { universidades u otros centros de formación y se cree que } \\
\text { sus gestores tienen dificultad y limitaciones para este tipo } \\
\text { de aprendizaje. }\end{array}$ & $\begin{array}{l}\text { Hall y Williams, (2008); Hall, } \\
\text { (2009a); Hjalager, (2002, 2010, } \\
\text { 2012); Alsos, et al., (2014) }\end{array}$ \\
\hline $\begin{array}{l}\text { Destinos turísticos exitosos y muy innovadores han desarro- } \\
\text { llado estructuras sin conocimientos previos. Para ello es fun- } \\
\text { damental el diseño y aplicación de políticas, donde el estado } \\
\text { en su papel legislador, planificador, coordinador y regulador } \\
\text { aplica estrategias integradoras e inclusivas, con patrocinios, } \\
\text { estímulos, promoción y protección de bienes culturales. }\end{array}$ & $\begin{array}{l}\text { Decelle, (2004); Hjalager et al., } \\
\text { (2008); Hall y Williams, (2008) }\end{array}$ \\
\hline $\begin{array}{l}\text { Sistemas de innovación local y regional, articulados al } \\
\text { nacional coadyuvan la distribución y uso del conocimiento y } \\
\text { son clave en industria turística para fomentar el aprendizaje } \\
\text { y redes internacionales. }\end{array}$ & $\begin{array}{l}\text { Hall y Williams, (2008); Hjalager, } \\
(2010,2012) \text {; Weidenfeld y Hall, } \\
(2014) .\end{array}$ \\
\hline
\end{tabular}

Fuente: Elaboración propia.

\section{ETNOTURISMO OPORTUNIDAD O AMENAZA PARA COMUNIDADES INDÍGENAS}

En turismo étnico la falta de empatía es una característica mucho más evidente desde las últimas décadas del siglo XX. De hecho, al comparar y evaluar las perspectivas de los cuatro grupos de actores clave en etnoturismo: gobierno, empresarios, minorías étnicas y visitantes, aunque podría haber otros como las agencias de viajes y guías se identifican resistencia en la regulación del estado frente a la autonomía étnica, en el exotismo cultural frente a la modernidad, entre desarrollo económico frente a preservación de la cultura y la autenticidad frente mercantilización cultural. De hecho, los esfuerzos para equilibrar la preservación de la integridad cultural sobre mercantilización de productos y servicios turísticos llegan a generar tensiones ideológicas debido a que habitualmente el discurso en el sector es dominado por voces no indígenas que tienen motivaciones turísticas occidentales, exigua orientación a entendimientos de sostenibilidad a largo plazo no compatibles con los intereses de las comunidades étnicas y no existen o son limitados los datos estadísticos 
socioeconómicos para fundamentar la toma de decisiones, conllevyo a establecer directrices políticas ampliamente generalizadas. Adicionalmente, consideraciones sobre la naturaleza y la contingencia de subjetividades encuentran que la pobreza parece ser una amenaza directa a la integridad cultural o aculturación en los negocios y no se reconocen características dinámicas evolutivas, únicas y en gran medida impredecibles, donde el recurso humano es fundamental para crear su propia realidad. Estos típicos factores endémicos no pueden ser ignorados, por el contrario, demyan mejor comprensión para la planificación y gestión de esta actividad económica que requiere integración de conocimientos de aborígenes como actores principales con conocimientos transdisciplinarios a fin de mostrar mayor conciencia y consideración en una justificación socioeconómica, cultural, política y medioambiental. Todo lo anterior, se consideran problemáticas estructurales carentes de autoridad y autonomía que instan la intervención efectiva del estado, la academia y la sociedad.

Significa entonces que la práctica etnoturística demya la implementación de acciones que contribuyan a lograr un balance para ser sostenible en el tiempo. Se cree que el turismo ha contribuido positivamente al crecimiento económico, no obstante, el crecimiento económico puede facilitar el desarrollo del turismo. Es preciso no pensar esta actividad sin esa simbiosis entre sus actores donde la cultura se convierta en un elemento central de producción de valor de una manera dinámica e interactiva, en la cual se demuestre empatía, incluyendo, pero no limitándola a un encuentro moral, de justicias sociales, de comprensión intercultural, de noción de paz, lo cual puede funcionar para alcanzar un buen nivel de comprensión en las relaciones intersubjetiva y los modos de conocer para aumentar las capacidades morales y afectivas de los profesionales del sector.

El turismo étnico es un sector viable para el desarrollo y crecimiento económico. Son múltiples las oportunidades de ingresos alternativos relacionadas con la revalorización de las tradiciones y prácticas culturales, la visualización de objetos, el desempeño del arte y la cultura, lo cual, puede influir en las formas en que las personas se ven a sí mismos y perciben su identidad. De hecho, el turismo es una fuerza social poderosa que puede alcanzar importantes fines cuyo su práctica está libre del fundamentalismo neoliberal del mercado y en cambio se aprovecha para satisfacer los imperativos de desarrollo humano y el bien público en general. Por ello, los indígenas dedicados a la actividad etnoturística les corresponde posicionarse como líderes en el impulso de proyectos de investigación y sus resultados. Posiblemente esto puede ser más probable con la mayor presencia de ellos en formación académica e investigativa que fomenta el mejoramiento o desarrollo de capacidades organizacionales sistémicas y promueven el éxito socioeconómico, cultural, medioambiental y político, es decir, desarrollyo competencias, habilidades y destrezas que minimizan la incertidumbre y permiten mantenerse en el mercado, también para requerir posteriormente limitada intervención del gobierno. De esa manera, el modelo de negocio étnico puede ser reconocido como una forma común de la industria de servicios, un nicho de mercado aliado para atender mercados objetivos de filiales extranjeras interesadas en ampliar y diversificar productos y servicios con énfasis en la calidad en un contexto global que lo exige.

Esta visión de desarrollo puede ser posible mediante la interconectividad con enfoque de sostenibilidad de los sectores políticos, económicos, sociales, ecológicos y culturales del contexto local. Deben ser explícitos tres principios: la población local usa, controla 
y gestiona sus recursos; una distribución equitativa de beneficios; y con el imperativo de sostenibilidad ambiental y cultural. Ello al reconocer beneficios del mejoramiento del escenario etnoturístico para las personas indígenas. No es una labor ni fácil ni sencilla que le corresponde asumir a los actores del sector turístico, quienes deben tomar decisiones con base a su real situación, necesidades y características distintivas, con el apoyo de conocimientos de ancianos locales y dio relevancia a lo que constituye su patrimonio, tangible e intangible, En el marco de las perspectivas expuestas se sugiere aplicar los conceptos de innovación analizados en el apartado 2.3, para contribuir al mejoramiento del escenario de este sector económico.

En la tabla 4 se muestra una síntesis de los aspectos del exotismo étnico que constituyen amenaza u oportunidad en el desarrollo de la actividad etnoturística en entornos megadiversos. Se observa en esos planteamientos fallas sistémicas que poco favorecen la competitividad, productividad y sostenibilidad de organizaciones etnoturística. Ciertamente no tienen procesos de $\mathrm{I}+\mathrm{D}$, empero, poseen abundante recursos y bienes tangibles e intangibles, la base para su impulso.

Tabla 4

ETNOTURISMO AMENAZA U OPORTUNIDAD PARA ENTORNOS MEGADIVERSOS

\begin{tabular}{|c|c|c|}
\hline FACTOR & ESCENARIOS & AUTORES \\
\hline \multirow[b]{3}{*}{$\begin{array}{l}\text { REPRESENTA } \\
\text { AMENAZA } \\
\text { CUYO }\end{array}$} & $\begin{array}{l}\text { Necesita la empatía como fuerza en la lucha } \\
\text { contra la xenofobia, producto de influencias } \\
\text { neoliberales, neocoloniales, fundamentalismos } \\
\text { y militarismo, manifestada en actitudes intimi- } \\
\text { datorias, racistas y bullying a otras personas. }\end{array}$ & $\begin{array}{l}\text { Tucker, (2016); Schwarzin, } \\
\text { (2012); Pedwell, (2012b). }\end{array}$ \\
\hline & $\begin{array}{l}\text { Grupos mayoritarios dominan y manipulan la } \\
\text { actividad etnoturística, mercantilizan represen- } \\
\text { taciones culturales de grupos étnicos minorita- } \\
\text { rios, especialmente con fines económicos. }\end{array}$ & Yang, et al., (2016) \\
\hline & $\begin{array}{l}\text { Evidencia tensión ideológica por acciones no } \\
\text { compatibles que buscan la preservación de la } \\
\text { integridad cultural sobre mercantilización de } \\
\text { productos y servicios. Habitualmente el discurso } \\
\text { en el sector es dominado por voces no indígenas } \\
\text { que tienen motivaciones turísticas occidentales } \\
\text { y exigua orientación a sostenibilidad a largo } \\
\text { plazo, contrario a los intereses de comunidades } \\
\text { étnicas y no existen o son limitados los datos } \\
\text { estadísticos socioeconómicos para fundamentar } \\
\text { la toma de decisiones, conllevyo a establecer } \\
\text { directrices políticas ampliamente generalizadas. }\end{array}$ & $\begin{array}{l}\text { Heldt y Mirya, (2015); } \\
\text { Nielsen y Wilson, (2012); } \\
\text { Hill, (2011); Yang, (2011); } \\
\text { Yang y Wall, (2009). }\end{array}$ \\
\hline
\end{tabular}




\begin{tabular}{|c|c|c|}
\hline FACTOR & ESCENARIOS & AUTORES \\
\hline \multirow{2}{*}{$\begin{array}{l}\text { REPRESENTA } \\
\text { AMENAZA } \\
\text { CUYO }\end{array}$} & $\begin{array}{l}\text { La pobreza es vista como amenaza directa a la } \\
\text { integridad cultural o aculturación en los nego- } \\
\text { cios etnoturísticos. }\end{array}$ & Cannon, (2011). \\
\hline & $\begin{array}{l}\text { No se reconoce la existencia de problemas } \\
\text { estructurales carentes de autoridad y autonomía. }\end{array}$ & $\begin{array}{l}\text { De la Meza, (2016); } \\
\text { Ryan, (2002). }\end{array}$ \\
\hline \multirow{4}{*}{$\begin{array}{l}\text { REPRESENTA } \\
\text { OPORTUNIDAD } \\
\text { CUYO }\end{array}$} & $\begin{array}{l}\text { Se demuestra empatía entre actores, incluyendo, } \\
\text { pero no limitándola a un encuentro moral, de } \\
\text { justicias sociales, de comprensión intercultural, } \\
\text { de noción de paz. Es decir, hay buen nivel de } \\
\text { comprensión intersubjetiva que es base para la } \\
\text { transformación social positiva, reconociendo y } \\
\text { respetyo la subjetividad y la representación de } \\
\text { los demás sin jerarquías opresivas de poder a } \\
\text { través de las fronteras geopolíticas. }\end{array}$ & $\begin{array}{l}\text { Mostafanezhad y } \\
\text { Hannam, (2014a); } \\
\text { Moufakkir y Kelly, } \\
\text { (2010); Tomljenovic } \\
\text { (2010); Higgins- } \\
\text { Desbiolles, (2006) }\end{array}$ \\
\hline & $\begin{array}{l}\text { La evolución de la capacidad de organización } \\
\text { sistemática de comunidad étnica contribuye } \\
\text { positivamente al desarrollo y al crecimiento } \\
\text { económico y éstos, facilitan el desarrollo del } \\
\text { turismo con ingresos alternativos relativos a } \\
\text { revalorización de las tradiciones y prácticas } \\
\text { culturales, visualización de sus objetos, buen } \\
\text { desempeño del arte y la cultura, además, influye } \\
\text { en las formas en que las personas se ven a sí } \\
\text { mismos y perciben su identidad. }\end{array}$ & $\begin{array}{l}\text { Zhang,(2016); Chen, } \\
\text { et al.,(2016); Heldt y } \\
\text { Mirya, (2015). }\end{array}$ \\
\hline & $\begin{array}{l}\text { El etnoturismo se reconoce como un negocio } \\
\text { común de la industria de servicios, un nicho de } \\
\text { mercado aliado para atender mercados objetivos } \\
\text { de filiales extranjeras interesadas en inhibir la } \\
\text { competencia internacional, siendo los indígenas } \\
\text { los líderes en el impulso resultados de estos } \\
\text { proyectos. }\end{array}$ & $\begin{array}{l}\text { Cohen, et al., (2016); } \\
\text { Yerson, et al., (2008) } \\
\text {; Imanishi, (2007) }\end{array}$ \\
\hline & $\begin{array}{l}\text { Aplicyo tres principios explícitos: la población } \\
\text { local usa, controla y gestiona sus recursos; } \\
\text { distribución equitativa de beneficios; e } \\
\text { imperativo de sostenibilidad ambiental y } \\
\text { cultural. Para ello, corresponde a actores del } \\
\text { etnoturismo tomar decisiones con base a su } \\
\text { real situación, necesidades y características } \\
\text { distintivas, con el apoyo de conocimientos de } \\
\text { ancianos locales como componente central. }\end{array}$ & $\begin{array}{l}\text { Turner y Turner, } \\
(2012) \text {. }\end{array}$ \\
\hline
\end{tabular}

Fuente: Elaboración propia 
Problemas estructurales que carecen de autenticidad y autoridad, asimismo, acciones que no evidencian comportamientos éticos en el desarrollo del etnoturismo, relacionadas con la oferta de productos y servicios únicamente para satisfacer necesidades básicas de grupos, así como gobiernos y burgueses dominyo y manipulyo indígenas para su beneficio particular e incluso el fingir ante viajeros de ocio manifestaciones culturales propias de otra cultura, son propias de sociedades globalizadas que colocan en riesgo a destinos etnoturísticos y generan resistencia entre el estado y comunidades nativas. Por un lado, el primero implanta ineficientes procesos de regulación, preservación y protección de los bienes históricos y culturales, mientras las segundas, hacen valer su autonomía étnica. Ello es muestra la necesidad de cambios estructurales en el sistema. Procesos macroambientales y del contexto local son muestra de falta de empatía en la industria turística que acrecienta la preocupación de la población por el desequilibrio entre la preservación de la de la integridad cultural sobre mercantilización de productos y servicios en el turismo. Es clave que grupos de interés locales representados en el estado, la academia y la sociedad civil asuman el reto de buscar mecanismos distintivos que mejoren este escenario en las comunidades indígenas, como un factor determinante de su desarrollo y crecimiento económico.

El etnoturismo como actividad económica del mundo globalizado que sustenta a organizaciones familiares insta procesos fundamentados en gestión tecnológica e innovación. A los indígenas se les debería promover el desarrollo de capacidades que favorezcan realizar actividades más complejas, con las cuales lideren el cambio de su actual situación al poner en marcha proyectos de I+D. Con ello, eventualmente en la oferta de sus productos y servicios se tiene como resultado, distribución equitativa de beneficios con perspectiva de sostenibilidad, conciencia por el impacto de las decisiones tomadas, respeto, sentido de pertenencia y responsabilidad por los bienes culturales que les identifican. También, les posibilita el establecimiento de redes académicas y empresariales y la conformación de sistemas de innovación a nivel local, regional y nacional.

\section{CONCLUSIONES}

Este documento, una primera aproximación de su tipo al tema, analiza factores que potencialmente pueden ser una oportunidad o una amenaza derivada de la influencia de los procesos macroambientales y locales del actual entorno globalizado a lo que se conoce como turismo indígena, aborigen o etnoturismo en la industria turística, una de las principales industrias de la economía que se ha favorecido con los adelantos tecnológicos para la diversificación de productos y servicios. Se infiere que en el desarrollo de esta actividad económica existe desequilibrio entre el atractivo de la etnicidad con la preservación y conservación de la identidad de la población indígena, por lo que se sienten vulnerable y les genera preocupación que el gobierno, gryes y medianas empresas e incluso otros grupos étnicos y visitantes o viajeros de ocio moderno, muestren falta de empatía con sus bienes patrimoniales, considerados el principal insumo.

Aun cuyo existen muchos grupos de interés alrededor de las organizaciones etnoturísticas que pueden coadyuvar al fortalecimiento e incremento de habilidades y competencias para desarrollar otras capacidades con las que eventualmente realicen tareas más complejas, 
a través de ellos no fluye procesos de aprendizajes y conocimientos con los que se superen fallas sistémicas y sean más competitivas, productivas y sostenibles en el tiempo. Se requiere fortalecer y establecer vínculos de largo plazo en y entre instituciones y que en esos relacionamientos se produzca flujo de conocimiento que, integrados a los conocimientos de base permitan el desarrollo de capacidades para la gestión de tecnología y de innovación.

Son muchos los cuestionamientos que a futuro requieren mayor análisis, entre ellos: ¿Qué tipo de tecnología para la innovación se debe gestionar para que la adaptabilidad indígena no se pierda en los procesos comerciales? ¿Qué tipo de estudio se requiere para comprender mejor cómo funcionan y se estructuran procesos para la gestión de transferencia tecnológica o de innovación en estos sistemas sociales, a partir del patrimonio tangible e intangible? ¿Qué tipo de valor genera la identidad cultural en la actividad etnoturística? ¿Cómo orientar un plan prospectivo de gestión de tecnología para la innovación en etnoturismo?

Todo lo expuesto anteriormente implica acciones políticas para el fortalecimiento de la actividad etnoturística. Comunidades étnicas con los grupos de interés del etnoturismo tienen en deber de protagonizar y asumir actitud proactiva para el establecimiento de relaciones de integración, inclusión y cooperación en y entre instituciones, para la conformación de redes de aprendizaje y de conocimiento articuladas a un eficiente sistema de innovación y, para la promoción de eficientes programas de difusión de información que creen conciencia en la humanidad de la importancia de la preservación y conservación del patrimonio cultural tangible e intangible, con ello, se busca crear mayor afinidad entre viajeros y nativos en el presente, también para que las futuras generaciones propendan la valoración y relevancia de la existencia del equilibrio de destinos etnoturísticos, aunado a esto, los organismos gubernamentales deberían realizar un mayor esfuerzo para obtener diagnósticos precisos y detallados de las capacidades y necesidades de las organizaciones etnoturística y datos estadísticos socioeconómico de jurisdicciones étnicas para el diseño e implantación de políticas públicas en este sector.

\section{AGRADECIMIENTOS}

Expresiones de total gratitud a la Universidad de La Guajira, Riohacha, Colombia, por el apoyo financiero para adelantar el proceso de formación en el programa Doctorado en Gestión de la Tecnología y la Innovación ofertado por la Universidad Pontificia (UPB), Medellín, Colombia. Desde ahí, con la orientación del comité tutorial de investigación está más cerca la posibilidad de hacer un aporte científico a la industria turística, específicamente, al desarrollo de la actividad etnoturística.

\section{REFERENCIAS}

AITCHISON, C. (2007): «Marking difference or making a difference: Constructing places, policies y knowledge of inclusion, exclusion y social justice in leisure, sport y tourism», en The critical turn in tourism studies: Innovative research methods. Oxford, USA. Elsevier, pp. 77-90. 
ALSOS, G.A., EIDE, D. y MADSEN, E.L. (Eds.) (2014): «Introduction: innovation in tourism industries», en Hybook of Research on Innovation in Tourism Industries. USA, Edward Elgar Publishing, pp. 1-24.

ÁVILA, J.C. (2012): «La doble dimensión de una capacidad organizacional: evidencias de una organización sin ánimo de lucro que compite en el mercado», Cuadernos de Administración, vol. 25 (44), pp.11-37.

BOISSEVAIN, J. (1996): Coping with tourists: European reactions to mass tourism. Oxford, USA. Berghahn Books (Vol. 1).

BLICHFELDT, B.S. (2009): «Innovation y entrepreneurship in tourism: The case of a Danish caravan site», Pasos. Revista de Turismo y Patrimonio Cultural, vol. 7 (3), pp. 415-431.

BUNTEN, A.C. (2008): «Sharing culture or selling out? Developing the commodified persona in the heritage industry», Journal of the American Ethnological Society, vol. 35 (3), pp. 380-395.

CAMISÓN, C. y FORÉS, B. (2010): «Knowledge absorptive capacity: New insights for its conceptualization y measurement», Journal of Business Research, vol. 63 (7), pp. 707-715.

CASTRO SPILA, J. et al. (2009): «Capacidad de absorción y formas de aprendizaje para la innovación: un modelo conceptual», en Projectics / Proyéctica / Projectique. De Boeck Supérieur, vol. 1, pp. 63-76.

CATON, K. (2012): «Thinking inside the box: Understying discursive production y consumption in tourism», en The critical turn in tourism studies: Creating an academy of hope. Routledge, London y New York, pp. 121-134.

CATON, K. (2014): «Humanism y tourism: A moral encounter», en Moral encounters in tourism. USA, Ashgate Publishing Limited, pp. 185-198.

CEBALlOS, H. (1998): Ecoturismo, Naturaleza y Desarrollo Sostenible. México, Editorial Diana.

CEPAL. (2014): Los pueblos indígenas en América Latina. Centro Latinoamericano y Caribeño de Demografía. CELADE.

COHEN, E. (1987): «Alternative Tourism: A critique», Tourism Recreation Research, vol. 12 (2), pp. 13-18.

COHEN, E. (1988): «Authenticity y commoditization in tourism», Annals of Tourism Research, vol. 15 (39), pp. 371-386.

COHEN, W. M. y LEVINTHAL, D.A. (1989): «Innovation y learning: the two faces of R \& D», The economic journal, vol. 99 (397), pp. 569596.

COHEN, W. y LEVINTHAL, D.A. (1990): «Absorptive capacity: A New Perspective on Learning y Innovation», in Administrative Science Quarterly, Special Issue: Technology, Organizations, y Innovation, vol. 35 (1), pp. 128-152.

COX, ARANIBAR R. (2009): Turismo indígena y comunitario en Bolivia. Un instrumento para el desarrollo socioeconómico e intercultural. La Paz, Bolivia, Editorial Plural.

DECELLE, X. (2004): A conceptual y dynamic approach to innovation in tourism. Maître de Conférences, Institut de Recherches et d'Etudes Supérieures du Tourisme (Irest) Université Paris 1 Panthéon-Sorbonne, France, OCDE. 
DYER, P., ABERDEEN, L. y SCHULER, S. (2003). «Tourism impacts on an Australian indigenous community: A Djabugay case study», Tourism Management, n²4(1), pp.83-95.

GET, D. y JAMIESON, W. (1997): «Rural tourism in Canada: issues, opportunities y entrepreneurship in aboriginal tourism in Alberta». In The business of rural tourism: International perspectives Toronto, International Thomson Business Press, pp. 93-107.

GRABURN, N.H. (1984): «The evolution of tourist arts», Annals of Tourism Research, vol. 11 (3), pp. 393-419.

HALL, C.M. (2009): «Innovation y tourism policy in Australia y New Zealy: Never the twain shall meet? », Journal of Policy Research in Tourism, Leisure y Events, vol. 1, pp. 2-18.

HALL, C.M. (2014): Tourism y social marketing. London y New York, Routledge Critical Studies in Tourism, Business y Management.

HALL, C.M. y WILLIAMS, A.M., (2008): Tourism y Innovation. London y New York, Routledge Contemporary Geographies of Leisure, Tourism y Mobility.

HELDT C. S. y MIRYA M.T. (2015): «Performing identity y culture in Indigenous tourism - a study of Indigenous communities in Québec, Canada», Journal of Tourism y Cultural Change, $\mathrm{n}^{\circ} 15$ (1), pp. 1-14.

HENDERSON, J. (2003): «Ethnic Heritage as a Tourist Attraction: The Peranakans of Singapore», International Journal of Heritage Studies, vol. 9 (1), pp. 27-44.

HIGGINS-DESBIOLLES, F. (2006): «More than an "industry": The forgotten power of tourism as a social force», Tourism Management, vol. 27 (6), pp. 1.192-1.208.

HJALAGER, A.M. (2002): «Repairing innovation defectiveness in tourism», Tourism Management, vol. 23 (5), pp. 465-474.

HJALAGER, A.M. (2010): «A review of innovation research on tourism», Tourism Management, vol. 31 (1), pp. 1-12.

HJALAGER, A.M. (2012): «Innovation policies for tourism» International Journal of Tourism Policy, vol. 4 (4), pp. 336-355.

HORNG, J.-S., LIU, C.-H., CHOU, H.-Y. y TSAI, C.-Y. (2012): «Understying the impact of culinary bry equity y destination familiarity on travel intentions», Tourism Management, vol. 33 (4), pp. 815-824.

IBÁÑEZ, J. (2005): «Diagnosis y Propuestas. TURISMO: Concepto y Estrategias para un Modelo, Formulación y Planificación», En Plan Territorial Especial de Ordenación de Actividad Turística. Isla La Palma, Islas Canarias, Archipiélago español, pp. 146-414.

IMANISHI, T. (2007): «An ethnic model of Japanese overseas tourism companies», Annals of Tourism Research, vol. 34 (2), pp. 517-536.

JIM, C.Y. (2000): «Environmental changes associated with mass urban tourism y nature tourism development in Hong Kong», The Environmentalist, vol. 20 (3), pp. 233-247.

KVAM, G. y STRÆTE, E.P. (2010): «Innovation y diffusion different roles in developing nature-based tourism», The Open Social Science Journal, vol. 3, pp. 30-40.

LARA, H. (2011): «Indigenous culture: both malleable y valuable», Journal of Cultural Heritage Management y Sustainable Development, vol. 1 (2) pp. 122-134. 
LEAL G. N. (2008): «Patrimonio cultural indígena y su reconocimiento institucional», Departamento de Ciencias Humanas, Facultad Experimental de Ciencias. Universidad del Zulia, $\mathrm{n}^{\circ} 24$ (56), pp. 28-43.

LUNDVALL, B. (2007): «National Innovation Systems-Analytical Concept y Development tool», Journal Industry y Innovation, vol.14 (1), pp.95-119.

MCINTOSH, A. J. y JOHNSON, H. (2005): «Exploring the nature of the Maori experience in New Zealy: Views from hosts y tourists», Journal Tourism, vol. 52 (2), pp. 117-129.

MCKERCHER, B. (2002): «Towards a classification of cultural tourists», International Journal of Tourism Research, vol. 4 (1), pp. 29-38.

MOSCARDO, G. (2008): «Sustainable Tourism Innovation: Challenging Basic Assumptions», Tourism y Hospitality Research, vol. 8 (1), pp. 4-13.

MIKKELSEN, C. (2015): El Mundo Indígena. Perú, Depósito Legal en la Biblioteca Nacional.

MOSTAFANEZHAD, M. y HANNAM, K. (2014): Moral Encounters in Tourism. Farnham, Surrey, Engly. Current Developments in the Geographies of Leisure y Tourism. Ashgate Publishing Ltd.

MOUFAKKIR, O. y KELLY, I. (Eds.). (2010): Tourism, Progress y Peace. Wallingford, Connecticut, USA. CABI International.

NIELSEN, N. y WILSON, E. (2012): «From Invisible to Indigenous-Driven: A Critical Typology of Research in Indigenous Tourism», Journal of Hospitality y Tourism Management, 19 (1), pp. 67-75.

OCDE (2001): Innovative clusters: Drivers of national innovation systems. Paris: OCDE OCDE (2006): Innovation y growth in tourism. Paris: OCDE.

OCDE (2009): Policy responses to the economic crisis: Investing in innovation for longterm growth. Paris.

OCDE (2012): Policy framework for policy coherence for development. Paris: OCDE.

OMT (2006): Reducción de la Pobreza por medio del Turismo; una compilación de buenas prácticas. Madrid, España.

PEDWELL, C. (2012): «Affective (self-) transformations: Empathy, neoliberalism y international development», Feminist Theory, vol.13 (2), pp.163-179.

PIKKEMAAT, B. y PETERS, M. (2005): «Towards the Measurement of Innovation- A Pilot Study in the Small y Medium Sized Hotel Industry», Journal of Quality Assurance in Hospitality \& Tourism, vol. 6 (3-4), pp. 89-112.

PNUMA, Programa de las Naciones Unidas para el Medio Ambiente, (2015): Marco de 10 años de programas sobre consumo sostenible y producción. Secretaria PNUMA www.unep.org/10yfp.

PULIDO FERNÁNDEZ, J.I. (2007): «Sostenibilidad de los destinos turísticos: Una aproximación a su medición en España», en La actividad turística española en 2006, AECIT, Editorial Universitaria Ramón Areces, España.

RICHARDS, G. (2014): «Creativity y tourism in the city», Current Issues in Tourism, vol. 17 (2), pp. 119-144.

ROBLEDO, J. (2013): Introducción a Gestión de la Tecnología e Innovación. Universidad Nacional de Colombia, Medellín. 
RYAN, C. (2002): «Tourism y Cultural Proximity: Examples from New Zealy», Annals of Tourism Research, vol. 29 (4), pp. 952-971.

RYAN, C. (2005): «Introduction: Tourist-Host Nexus-Research Considerations», In Indigenous tourism: The commodification y management of culture. Advances in Tourism Research Series, Elsevier, Amsterdam the Netherlys, pp. 1-11.

RYAN, C. y AICKEN, M. (2005): Indigenous tourism: The commodification y management of culture. Amsterdam the Netherlys, Advances in Tourism Research Series, Elsevier.

SAKDIYAKORN, M. y SIVARAK, O. (2016): «Innovation Management in Cultural Heritage Tourism: Experience from the Amphawa Waterfront Community, Thaily», Journal of Tourism Research, vol. 21 (2), pp. 212-238

SCHWARZIN, L. (2012): «To act as though the future mattered: a framework for hopeful tourism education», en The critical turn in tourism studies: Creating an academy of hope. Routledge, London y New York, pp.134-148.

SMITH, V. (1977): [1989]. Hosts y guests: The anthropology of tourism. Philadelphia, University of Pennsylvania Press.

STAMBOULISA, Y. y SKAYANNIS, P. (2003): «Innovation strategies y technology for experience-based tourism», Tourism Management, vol. 24 (1), pp. 35-43.

STRONZA, A. y GORDILLO, J. (2008): «Community Views of Ecotourism», Annals of tourism research, vol. 35 (2), pp. 448-468.

TANG, X., (2016): «The historical evolution of China>s tourism development policies (1949-2013)-a quantitative research approach», Tourism Management, vol. 58 (33), pp.259-269.

THOMAS, R. y WOOD E. (2014): «Innovation in tourism: Re-conceptualising y measuring the absorptive capacity of the hotel sector», Tourism Management, vol. 45, pp. 39-48.

TOMLJENOVIC, R. (2010): «Tourism y Intercultural Understying or Contact Hypothesis Revisited», en Tourism, progresses y peace. Croatia, Institute for Tourism, pp. 17-34.

TUCKER, H. (2016): «Empathy y tourism: Limits y possibilities», Annals of Tourism Research, vol. 57, pp. 31-43.

UNESCO (2009): Lista Representativa del Patrimonio Cultural Inmaterial de la Humanidad. Convención para la Salvaguardia del Patrimonio Cultural Inmaterial de 2003.

WALSH, E. y SWAIN, M. (2004): «Creating Modernity by Touring Paradise: Domestic Ethnic Tourism in Yunnan, China», Tourism Recreation Research, vol. 29 (2), pp. 59-68.

WEIERMAIR, K. (2006): «Product improvement or innovation: what is the key to success in tourism? », In OCDE. (Ed.), Innovation y Growth in Tourism, Paris: OECD, pp. 53-69.

WEIDENFELD, A. y HALL, C. M. (2014): «Tourism in the Development of Regional y Sectoral Innovation Systems», en The Wiley Blackwell Companion to Tourism, Oxford, pp. 578-588.

WHEELEN, T y HUNGER, D. (2012): Administración estratégica y política de negocio. Conceptos y casos. México, Pearson Educación. 
XIANG. Y.M., ARCODIA, C. y RUHANEN, L. (2013): «The national government as the facilitator of tourism innovation: evidence from Norway», Journal Current Issues in Tourism, vol. 18 (12), pp. 1172-1191.

YANG, J., RYAN, C. y ZHANG, L. (2014): «Sustaining culture y seeking a Just Destination: governments, power y tension -a life- cycle approach to analyzing tourism development in an ethnic inhabited scenic area in Xinjiang, China», Journal of Sustainable Tourism, vol. 22 (8), pp.1151-1174.

YANG, J., RYAN, C. y ZHANG, L. (2016): «Impersonation in ethnic tourism-the presentation of culture by other ethnic groups», Annals of Tourism Research, vol. 56, pp.16-31.

YANG, L. y WALL, G. (2009): «Ethnic tourism: A framework y an application», Tourism Management, vol. 30 (4), pp. 559-570.

YANG, L. WALL, G. y SMITH, S. (2008): «Ethnic tourism development: Chinese Government Perspectives», Annals of Tourism Research, vol. 35 (3), pp.751-771.

YANG, Y. y FIK, T. (2014): «Spatial effects in regional tourism growth», Annals of Tourism Research, vol. 46, pp. 144-162.

YANG, Y., y WONG, K. K. (2012): «A Spatial Econometric Approach to Model Spillover Effects in Tourism Flows», Journal of Travel Research, vol. 51 (6), pp. 768-778.

YERSON, C, BUNDA, T. y WALTER, M. (2008): «Indigenous higher education: The role of universities in releasing the potential», The Australian Journal of Indigenous Education, vol. 37 (1), pp.1-8

ZAHRA, S.A. y GEORGE, G. (2002): «Absorptive Capacity: A Review, Reconceptualization y Extension», Academy of Management Review, vol. 27 (2), pp. 185-203.

ZHANG, J. (2016): «Weighing y realizing the environmental, economic y social goals of tourism development using an analytic network process-goal programming approach», Journal of Cleaner Production, vol. 127, pp. 262-273.

ZHANG, A.L., PHUR, S. y DEKYI, Y. (2013): «The empirical analysis on the economic impact of developing tourism industry in Tibet», AL Zhang, BZM Pu, JYZ Journal of Tibet University, Social Science Edition, vol. 28 (44), pp. 18-25.

ZHANG, C. y XIAO, C. (2014): «Destination development in China: towards an effective model of explanation», Journal of Sustainable Tourism, vol. 22 (2), pp. 214-233. 